\title{
Neurocognitive and Quality-of-Life Outcomes Following Intensive Care Admission: A Prospective 6-Month Follow-Up Study
}

Viswesvaran Balasubramanian ${ }^{1}$, Jagdish C Suri ${ }^{2}$, Pranav Ish ${ }^{3}$, Nitesh Gupta ${ }^{4}$, Debasis Behera ${ }^{5}$, Pankaj Gupta ${ }^{6}$, Shibdas Chakrabarti ${ }^{7}$

\begin{abstract}
Background: Post-intensive care survivors have decreased quality of life scores and prolonged cognitive dysfunction due to baseline factors and events related to intensive care unit admission, which remain largely unrecognized.

Materials and methods: A prospective observational cohort study to assess the quality of life and occurrence of cognitive dysfunction, 3 and 6 months following discharge from the intensive care unit, was carried out. We enrolled 136 adults presenting to the intensive care unit with no prior cognitive dysfunction or depression and followed up and assessed them with repeatable battery for the assessment of neuropsychological status (RBANS) and quality of life with short Form-36 (SF-36) health survey.

Results: The incidence and prevalence of cognitive dysfunction was $100 \%$ at 3 and 6 months, respectively, as assessed by RBANS with a global cognition scores at 3 and 6 months of 71 (IQR 68.5-73) and 74 (IQR 72-86), respectively. Higher Charlson's comorbidity score, increased severity of illness, longer duration of mechanical ventilation, pain, delirium, coma, and hospital stay were associated with statistically significant lower scores at 3 months. The median SF-36 mental component score (MCS) and physical component score (PCS) at 3 months were 38.4 and 32.5 and at 6 months were 38.2 and 32.6, respectively. Poor score was associated significantly with advancing age, poor functional parameters at baseline as evidenced by clinical frailty, poor baseline Katz ADL scores, increased severity of illness, longer duration of mechanical ventilation, occurrence and duration of delirium, coma, pain, and usage of sedatives with or without analgesics.

Conclusion and clinical significance: Patients discharged from the intensive care unit are at high risk for persistent cognitive impairment and poor quality of life score. Poor baseline patient characteristics and events occurring in ICU are associated with worse cognition and quality of life scores. There is an urgent need to prevent, diagnose, and manage these patients by optimizing intensive care practices.

Keywords: Neurocognitive impairment, Post intensive care, Quality of life.

Indian Journal of Critical Care Medicine (2020): 10.5005/jp-journals-10071-23576
\end{abstract}

\section{INTRODUCTION}

Advances in medical technology and therapeutics have reduced mortality rates and extended lives of critically ill patients. Millions of patients who survive critical illnesses each year are burdened with acquired impairment in cognition, mental health, and/or functional disability, which remains largely underrecognized. ${ }^{1}$ While some patients do return back to their precritical illness level of health and functional status functioning, many patients experience impairments in mental health, cognition, physical health, and quality of life. ${ }^{2}$

Critical illness survivors can have a persistent and often underestimated cognitive dysfunction, ${ }^{3-5}$ which is characterized by fresh deficits or worsening of preexisting deficits in cognition and/or executive function. This long-term cognitive impairment postcritical illness is an emerging public health issue, given the enormous number of acutely ill patients who are under treatment in intensive care units (ICUs). ${ }^{6}$ Among elderly, cognitive decline is associated with prolonged hospitalization also., ${ }^{7,8}$

Understanding the epidemiology and risk factors is essential as the subsequent interventions should be initiated at the earliest to prevent the cognitive impairment, improve mental, physical health and quality of life, and reduce the long-term morbidity and mortality rates of ICU survivors. We conducted a prospective observational cohort study to evaluate the quality of life and

\footnotetext{
${ }^{1-7}$ Department of Pulmonary, Critical Care and Sleep Medicine, Vardhman Mahavir Medical College and Safdarjung Hospital, New Delhi, India

Corresponding Author: Shibdas Chakrabarti, Department of Pulmonary, Critical Care and Sleep Medicine, Vardhman Mahavir Medical College and Safdarjung Hospital, New Delhi, India, Phone: +91 9968303547, e-mail: pccm.sjh@gmail.com

How to cite this article: Balasubramanian V, Suri JC, Ish P, Gupta N, Behera D, Gupta P, et al. Neurocognitive and Quality-of-Life Outcomes Following Intensive Care Admission: A Prospective 6-Month Follow-Up Study. Indian J Crit Care Med 2020;24(10):932-937.

Source of support: Nil

Conflict of interest: None
}

occurrence of cognitive dysfunction, 3 and 6 months following discharge from ICU.

\section{Materials and Methods}

The study was conducted in the ICU of a tertiary care center over a time period of 18 months. All adult patients above 18 years of age presenting to ICU with respiratory failure, septic shock or cardiogenic shock, and consent for follow-up were included in the 
study. We excluded individuals with a past history of ICU exposure in recent times (i.e., mechanical ventilation in the preceding 2 months before the current admission, $>5$ ICU days stay in the month before the present ICU admission); patients who could not be reliably assessed for delirium owing to deafness and blindness; and patients at risk for preexisting cognitive deficits due to neurodegenerative disease, severe dementia, suspected anoxic brain injury, or out-ofhospital cardiac arrest. We also excluded patients with score $>3.3$ in Short Informant Questionnaire on Cognitive Decline in the Elderly $(\mathrm{IQCODE})^{9}$ during screening for preexisting cognitive impairment in patients $>50$ years of age and for patients $<50$ years but with known memory disorders. An informed consent was obtained from patients or next to kin. If consent was initially obtained from next to kin, then informed consent was obtained from the patient when he/she was competent. The questionnaires were administered by the intensivist in the patient's or next to kin's own language of understanding as and when needed. The study was approved by the institutional ethics committee. All the questionnaires were administered by the intensivist with translation into Hindi as and when needed.

We obtained data on baseline demographics, history of depression, preexisting cognitive impairment, and other mental health illnesses as told by patient or next to kin (including psychiatric conditions diagnosed previously by a healthcare professional), education, activities of daily living (ADL), and instrumental ADL (IADL) at the time of enrolment with the Katz ADL Scale ${ }^{10}$ and Pfeffer functional activities questionnaire (PFAQ) questionnaires ${ }^{11}$ (though a healthcare proxy), clinical frailty score (CFS), ${ }^{12}$ illness severity according to the acute physiology and chronic health evaluation (APACHE) II score, sequential organ failure assessment (SOFA) score, ${ }^{13}$ comorbidities according to the Charlson comorbidity index $(\mathrm{CCl}),{ }^{14}$ and admission diagnosis. Up to the entire duration of stay in hospital, we evaluated the patients twice a day in the ICU and once a day in the wards for the level of consciousness using the Richmond agitation-sedation scale (RASS) ${ }^{15}$ and for delirium with the confusion assessment method for the ICU (CAM-ICU). ${ }^{16}$ Patients were considered to be delirious if they were responding to verbal stimuli (RASS score value -3 or more) and CAM-ICU positive. They were considered comatose if they were not responding to verbal stimuli (RASS score value of -4 or -5 ). For patients in ICU, SOFA score was calculated every day and the use of sedatives and analgesics was documented from the medication records for entire duration of hospital stay.

At 3 and 6 months after hospital discharge (with a leeway of 15 days allowed on both sides of the target date), patients were assessed for cognitive dysfunction with the RBANS Update questionnaire ${ }^{17}$ and quality of life with the Short Form-36 (SF-36) Health Survey-Mental Component Score (MCS) and Physical Component Score (PCS) questionnaire. ${ }^{18}$

\section{Statistical Analysis}

All reported confidence intervals were two-sided with a $p$ value of less than 0.05 considered and recorded significant. Continuous variables in the study were expressed as median and interquartile range, with correlation between qualitative data was performed using the Pearson Chi-square test and Fisher's exact test. Correlation between qualitative and quantitative data was performed using the Kruskal-Wallis test and Mann-Whitney test. Correlation between two quantitative variables was performed using the Spearman's rho test. The analysis was performed using the SPSS (Statistical Package for the Social Sciences) software (IBM Corp. Released 2013. IBM SPSS Statistics for Windows, Version 22.0. Armonk, NY: IBM).

\section{Results}

Over a span of 18 months, 136 patients were enrolled of which 18 (13.2\%) patients died prior and 14 patients (10.3\%) did not return for follow-up at 3 months. Of the 104 patients followed up after 3 months, 19 (18.3\%) patients died and 10 (9.61\%) patients did not return to follow-up and a total of 75 patients had complete data at the end of 6 months. The 136 patients enrolled in the study had a median age of 56 years (IQR 49.2-65.7) at baseline. None of the patients having preexisting or past cognitive impairment as assessed by short IQCODE ${ }^{9}$ (score of 3.6 or greater) or by medical history of depression in the past as told by patient's attender was enrolled. Baseline characteristics of cohort at baseline, 3-month, and 6-month follow-up are given in Table 1. Delirium was observed in $70(51.5 \%)$ of 136 patients during the period of hospital stay with a median duration of 2 days. Data regarding the cognitive and QOL outcomes at 3 and 6 months' follow-up are given in Table 2.

Median RBANS global cognition scores recorded at 3 and 6 months were 71 (IQR 68.5-73) and 74 (IQR 72-86), respectively. The median scores for individual domains of cognitive were low in comparison to age-adjusted population mean (100). Thus, the incidence and prevalence of cognitive dysfunction was $100 \%$ at 3 and 6 months', respectively, as assessed by RBANS update. Median scores were lower in patients with age $>65$ years at 3 and 6 months (Fig. 1). Higher Charlson's comorbidity score, increased severity of illness as evidenced by higher APACHE and SOFA scores, longer duration of mechanical ventilation, pain, delirium, coma, and hospital stay were associated with statistically significant lower RBANS global cognition scores at 3 months. All the above parameters except for longer duration of mechanical ventilation, baseline APACHE and SOFA scores were associated with poorer cognitive scores at 6 months (Table 3).

The median SF-36 mental component score (MCS) and physical component score (PCS) at 3 months were 38.4 and 32.5 and at 6 months were 38.2 and 32.6 , respectively.

Poor SF-36 Mental component score at 3 and 6 months was associated significantly with advancing age, poor functional parameters at baseline as evidenced by clinical frailty, poor baseline Katz ADL scores, increased severity of illness as evidenced by higher APACHE and SOFA scores, longer duration of mechanical ventilation, occurrence and duration of delirium, coma, pain, and usage of sedatives with or without analgesics. In addition, poor SF-36 MCS at 6 months was also found to be associated with poor baseline PFAQ scores.

The SF-36 physical component score at 3 and 6 months was found to be associated with advanced age, clinical frailty, baseline poor Katz score and PFAQ, and higher Charlson comorbidity index. In addition, poor SF-36 (PCS) at 6 months was also found to be associated with longer duration of hospital stay (Table 3).

\section{Discussion}

To the best of our review and knowledge, this study is one of the few studies undertaken in Indian population to assess the risk factors associated with cognitive dysfunction and poor quality of life scores following discharge from the ICU. Though incidence, risk factors, prevalence, and outcome of delirium were studied in Indian population, ${ }^{19}$ the influence of such events in mental 
Table 1: The baseline characteristics of the patients in hospital and in the cohorts of follow-up

\begin{tabular}{|c|c|c|c|}
\hline Characteristic & $\begin{array}{l}\text { In-hospital cohort } \\
(n=136)\end{array}$ & $\begin{array}{l}\text { Follow-up cohort at } 3 \text { months } \\
(n=104)\end{array}$ & $\begin{array}{l}\text { Follow-up cohort at } 6 \text { months } \\
(n=75)\end{array}$ \\
\hline Age-median (IQR) & 56 years $(49.2-65.7)$ & 55 years $(49-64.75)$ & 54 years $(46-59)$ \\
\hline Male-number (\%) & $90(66.2 \%)$ & $74(71.2 \%)$ & $53(70.7 \%)$ \\
\hline $\mathrm{CCl}$-median (IQR) & $3(1-5)$ & $3(1-5)$ & $2(1-4)$ \\
\hline APACHE-median (IQR) & $25.5(22-28)$ & $24(20-26)$ & $22(19-24)$ \\
\hline SOFA—median (IQR) & $12(10-14)$ & $11(10-13)$ & $11(9-12)$ \\
\hline \multicolumn{4}{|l|}{ Diagnosis—no (\%) } \\
\hline Sepsis & $16(12 \%)$ & $5(4.8 \%)$ & $4(5.3 \%)$ \\
\hline ARDS & $21(15.4 \%)$ & $12(11.5 \%)$ & $6(8 \%)$ \\
\hline AECOPD & $32(23.5 \%)$ & $29(27.9 \%)$ & $26(34.7 \%)$ \\
\hline Asthma & $7(5.1 \%)$ & $5(4.8 \%)$ & $5(6.7 \%)$ \\
\hline Pulmonary edema & $19(14 \%)$ & $15(14.5 \%)$ & $10(13.5)$ \\
\hline Pulmonary embolism & $4(2.9 \%)$ & $3(2.9 \%)$ & $2(2.6 \%)$ \\
\hline Interstitial lung disease & $3(2.2 \%)$ & $3(2.9 \%)$ & $2(2.6 \%)$ \\
\hline Post-tubercular sequalae & $23(16.9 \%)$ & $22(21.1 \%)$ & $16(21.3 \%)$ \\
\hline Obstructive sleep apnea & $7(5.1 \%)$ & $7(6.7 \%)$ & $4(5.3 \%)$ \\
\hline Cardiogenic & $4(2.9 \%)$ & $3(2.9 \%)$ & 0 \\
\hline Duration of hospital stay-median (IQR) & 12 days $(8-14)$ & 10 days $(7.25-12)$ & 9 days $(6-12)$ \\
\hline Mechanical ventilation ( $\mathrm{n} \%)$ & $122(89.7 \%)$ & $97(93.2 \%)$ & $69(92 \%)$ \\
\hline Median duration (IQR) & 6 days $(4-8)$ & 5 days $(4-8)$ & 4 days $(3-7)$ \\
\hline Delirium—no (\%) & $70(51.5 \%)$ & $44(42.3 \%)$ & $27(36 \%)$ \\
\hline Median duration (IQR) & 2 days $(0-3)$ & 0 days $(0-2)$ & 0 days $(0-2)$ \\
\hline Coma-no (\%) & $49(36 \%)$ & $32(30.8 \%)$ & $16(21.3 \%)$ \\
\hline Median duration (IQR) & 0 days $(0-1)$ & 0 days $(0-1)$ & 0 days $(0-0)$ \\
\hline \multicolumn{4}{|l|}{ Use of sedative or analgesic agents—no (\%) } \\
\hline Benzodiazepine & $52(38.2 \%)$ & $32(30.7 \%)$ & $16(11.8 \%)$ \\
\hline Morphine & $12(8.8 \%)$ & $5(3.7 \%)$ & $4(5.3 \%)$ \\
\hline Fentanyl & $51(37.5 \%)$ & $32(30.7 \%)$ & $16(11.8 \%)$ \\
\hline Dexmedetomidine & $5(3.7 \%)$ & $1(0.9 \%)$ & $1(1.3 \%)$ \\
\hline \multicolumn{4}{|l|}{ History of ADL disability } \\
\hline Full function & $68(50 \%)$ & 55 (52.9\%) & $50(66.7 \%)$ \\
\hline Moderate impairment & $68(50 \%)$ & $49(47.1 \%)$ & $25(33.3 \%)$ \\
\hline Severe impairment & 0 & 0 & 0 \\
\hline \multicolumn{4}{|l|}{ PFAQ } \\
\hline Impairment & $68(50 \%)$ & $49(47.1 \%)$ & $25(33.3 \%)$ \\
\hline No impairment & $68(50 \%)$ & $55(52.9 \%)$ & $50(66.7 \%)$ \\
\hline PFAQ_-median score (IQR) & $10.5(8-15)$ & $9(8-15)$ & $9(8-13)$ \\
\hline \multicolumn{4}{|l|}{ Clinical frailty scale } \\
\hline Frail & $67(49.2 \%)$ & $49(47.1 \%)$ & $25(33.3 \%)$ \\
\hline Not frail & 69 & $55(52.9 \%)$ & $50(66.7 \%)$ \\
\hline Duration of pain-median (IQR) & 2 days $(1-5)$ & 1 days $(1-4)$ & 1 days $(0-4)$ \\
\hline
\end{tabular}

$\mathrm{IQR}$, interquartile range; $\mathrm{CCl}$, charlson comorbidity index; $\mathrm{APACHE}$, acute physiology and chronic health evaluation; SOFA, sequential organ failure assessment; ARDS, acute respiratory distress syndrome; AECOPD, acute exacerbation of chronic obstructive pulmonary disease; no, number; ADL, activities of daily living; PFAQ, Pfeffer functional activities questionnaire

health on a long-term basis of post-ICU survivors is unknown. The BRAIN-ICU cohort study analyzed the depression, long-term cognitive impairment, posttraumatic stress disorder, and functional disability in patients of critical illness discharged from ICU. ${ }^{20,21}$ However, the study was conducted in only U.S. population and hence the generalizability of the data to people of different racial and demographic backgrounds remains questionable. Hence, it was imperative to assess the long-term mental and physical health parameters in Indian population as we believe that early identification and intervention may help in improving the long-term outcomes of post-ICU survivors.

In the current study, cognition was affected in all patients of the follow-up cohort across all age groups. Since none of the patients with prior cognitive dysfunctions were included in this study, these 
Table 2: Long-term cognitive and QOL outcomes at 3 and 6 months

\begin{tabular}{lll}
\hline Outcome & At 3 months $(n=104)$ & At 6 months $(n=75)$ \\
\hline $\begin{array}{l}\text { Cognition } \\
\text { RBANS global cognition score }\end{array}$ & $71(68.5-73)$ & $74(72-76)$ \\
RBANS immediate memory score & $79.5(77-81.5)$ & $80(78-82)$ \\
RBANS delayed memory score & $82(80-84)$ & $84(82-86)$ \\
RBANS attention score & $80(78-82)$ & $84(82-86)$ \\
Quality of life [SF-36] & & \\
Mental component & 38.4 & 38.2 \\
Physical component & 32.5 & 32.6 \\
\hline
\end{tabular}

RBANS, repeatable battery for the assessment of neuropsychological Status; SF-36, short form survey 36. The RBANS is a brief, individually administered test measuring attention, language, visuospatial/constructional abilities, and immediate and delayed memory. It consists of 12 subtests, which yield 5 index scores and a total scale score. Index scores have a mean of 100 and standard deviation of 15 . SF-36 scores range from 0 to 100 . Higher scores indicate better quality of life
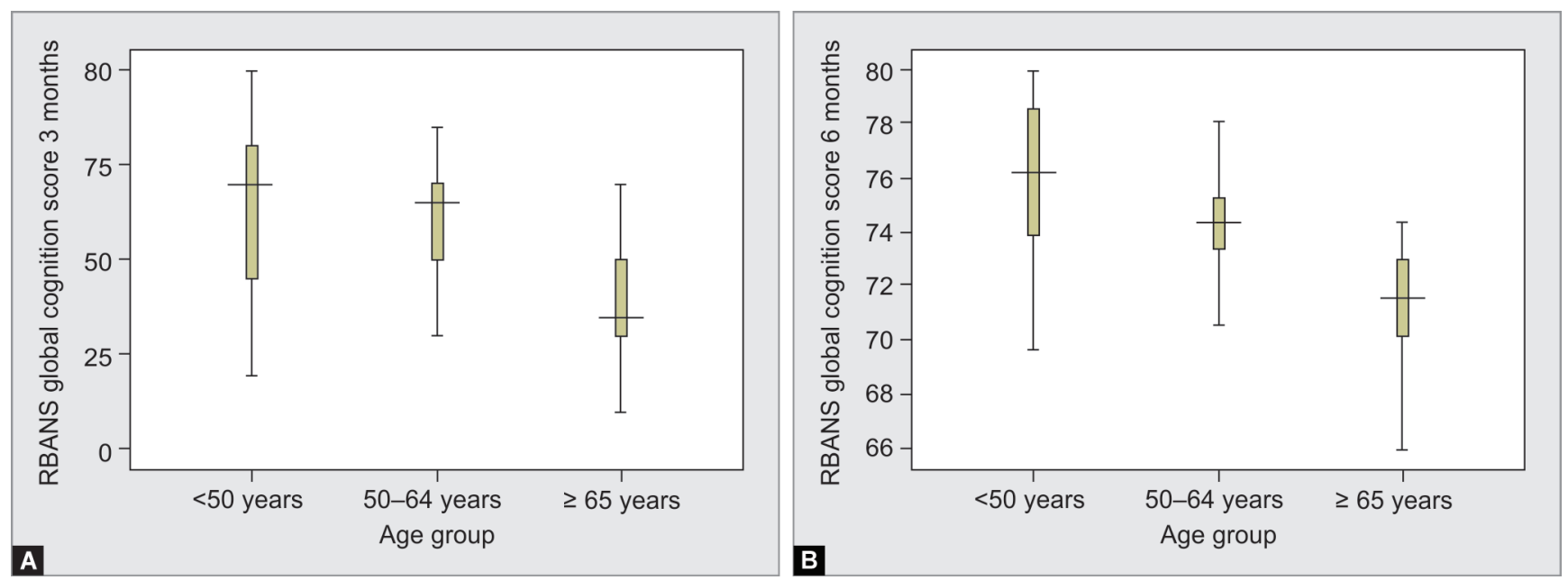

Figs $1 \mathrm{~A}$ and B: Box and whisker plot showing correlation between RBANS global cognition score and age in the cohort at 3 and 6 months

Table 3: Correlation of baseline parameters with outcome parameters at 3 and 6 months

\begin{tabular}{|c|c|c|c|c|c|c|}
\hline \multirow{2}{*}{$\begin{array}{l}\text { Baseline parameters } \\
\text { Gender }\end{array}$} & \multicolumn{2}{|c|}{$\begin{array}{c}\text { Age adjusted RBANS global cognition } \\
\text { score at } 3 \text { months } 6 \text { months }\end{array}$} & \multicolumn{2}{|c|}{$\begin{array}{c}\text { SF-36 (PCS) at } \\
3 \text { months } 6 \text { months }\end{array}$} & \multicolumn{2}{|c|}{$\begin{array}{c}\text { SF-36 (MCS) at } \\
3 \text { months } 6 \text { months }\end{array}$} \\
\hline & - & - & 0.469 & 0.549 & 0.343 & 0.343 \\
\hline Age & - & - & $<0.001$ & $<0.001$ & 0.019 & 0.019 \\
\hline Duration of mechanical ventilation & $<0.001$ (rho- 0.468$)$ & 0.114 (rho-0.184) & 0.383 & 0.383 & $<0.001$ & $<0.001$ \\
\hline Duration of delirium & $<0.001$ (rho-0.544) & $\mathbf{0 . 0 0 6}$ (rho-0.312) & 0.774 & 0.121 & $<0.001$ & $<0.001$ \\
\hline Duration of coma & $<\mathbf{0 . 0 0 1}$ (rho-0.425) & $\mathbf{0 . 0 1 2}$ (rho-0.260) & 0.159 & 0.102 & $<0.001$ & $<0.001$ \\
\hline Sedative \pm analgesia & - & - & 1.000 & 0.771 & $<0.001$ & $<0.001$ \\
\hline Clinical frailty score & - & - & 0.018 & $<0.001$ & 0.008 & 0.008 \\
\hline Baseline KATZ ADL score & - & - & $<0.001$ & $<0.001$ & 0.027 & 0.027 \\
\hline Baseline PFAQ score & - & - & $<0.001$ & $<0.001$ & 0.109 & $<0.05$ \\
\hline APACHE score & $<0.001$ (rho- 0.570 ) & 0.092 (rho-0.196) & 0.938 & 0.328 & $<0.001$ & $<0.001$ \\
\hline SOFA score & $<0.001$ (rho-0.512) & 0.092 (rho-0.196) & 0.369 & 0.194 & $<0.001$ & $<0.001$ \\
\hline Number of hospital days & $<\mathbf{0 . 0 0 1}$ (rho-0.675) & $\mathbf{0 . 0 0 9}$ (rho-0.300) & 0.142 & 0.018 & $<0.001$ & $<0.001$ \\
\hline Charlson Comorbidity Index & $<\mathbf{0 . 0 0 1}$ (rho-0.334) & $\mathbf{0 . 0 0 2}$ (rho- 0.352 ) & $<0.001$ & $<0.001$ & 0.249 & 0.249 \\
\hline Duration of pain & $<\mathbf{0 . 0 0 1}$ (rho-0.552) & $\mathbf{0 . 0 2 4}$ (rho- -0.260$)$ & 0.986 & 0.053 & $<0.001$ & $<0.001$ \\
\hline
\end{tabular}

RBANS, repeatable battery for the assessment of neuropsychological status; SF-36, short Form Survey 36; ADL, activities of daily living; PFAQ, Pfeffer functional activities questionnaire; APACHE, acute physiology and chronic health evaluation; SOFA, sequential organ failure assessment Bold values signify the $p$-value $<0.05$; It represents the statistically significant. Correlation of baseline parameters with outcome parameters

profound cognitive impairments were newly acquired. A systematic review observed the incidence of cognitive decline after critical illness to vary between 4 and $64 \%$ in adult patients evaluated and followed up to a time frame between 2 and 156 months after critical illness and attributed such highly variable incidence to varied definitions of cognitive impairment used in individual studies, 
different time frame of follow-up, failure to adjust for the pre-ICU cognitive function, and comorbidities. ${ }^{22}$

Though cognitive dysfunction was spread across all age groups and was more severe at 3 months, $32 \%$ of 6 -month follow-up had scores worse than those occurring in Alzheimer's disease indicating that events occurring in ICU can lead to long-term impact in patient's cognition. In our study, the univariate analysis revealed poor baseline factors such as higher Charlson's comorbidity score, higher APACHE and SOFA scores at presentation, longer duration of mechanical ventilation, pain, delirium, coma, and hospital stay to have a statistically significant association with lower RBANS global cognition scores at 3 months. However, duration of mechanical ventilation and APACHE and SOFA scores were not associated with significant poorer cognitive scores at 6 months. This implies that the cognition deficits occurring at 3 months secondary to initial severity of illness and mechanical ventilation might be reversible as evidenced by improved cognition scores at 6 months.

However, other parameters like poor baseline $\mathrm{CCl}$ scores, duration of pain, delirium, coma, and hospital stay had long-term impacts on cognitive functions. This was similar to the findings from systematic review, which found inconsistent or no associations of cognitive dysfunction with hypoglycemia, hyperglycemia, variations in serum glucose levels, in-hospital stay acute stress symptoms, mechanical ventilation, use of sedatives, analgesic or vasopressors medications, enteral feeding, extracorporeal membrane oxygenation, hypoxia, systolic blood pressure, pulse rate, or length of ICU stay. ${ }^{22}$ However, in the present study, poor baseline $\mathrm{CCl}$, duration of pain, coma, and length of hospital stay had impact on cognitive outcomes at 3 and 6 months.

In the present study, the presence and the duration of delirium was associated with worsening cognition scores at 3 and 6 months suggesting a long-term impact of delirium. Investigators in the BRAIN-ICU cohort also observed that the duration of delirium was associated with poor long-term global cognition and executive function and this association was independent of sedative or analgesic usage, age, preexisting cognitive impairment, the comorbidities, and other organ system failures during ICU care. ${ }^{20}$ Similar observations were also observed in studies involving acute respiratory distress syndrome (ARDS) survivors and post-critical care survivors where delirium developing during the intensive care unit stay had significantly more cognitive issues even after adjusting for various covariates. ${ }^{23}$ In addition, the duration of delirium was associated with long-term cognitive dysfunction. ${ }^{24}$

The association of delirium with poor cognitive functions is multifactorial and complex. Various neurotoxic, neuromodulatory, and neuroinflammatory mediators have been implicated. It is speculated that several factors including disturbed sleep, medications, hypoxia, and dysglycemia alter and affect the neurotransmitter production, action, and availability, which may have a role in psychological manifestations occurring during critical illness. ${ }^{25,26}$ Such neuroinflammation was observed to be precipitated by sepsis and $\operatorname{ARDS}^{27}$ due to an increased release of cytokines and reactive oxygen species, which affect the microglia and leads to synaptic and neuronal signal disruption. ${ }^{28,29}$ In addition to this, it may also be associated with structural changes like cerebral atrophy ${ }^{19}$ and reduced white-matter integrity. ${ }^{30} \mathrm{~A}$ prospective cohort study observed that patients with delirium of longer duration had a greater brain atrophy when evaluated with magnetic resonance imaging carried out 3 months after discharge besides worse cognitive function at 12 months' follow-up. ${ }^{3}$
Similarly, the median MCS and PCS of SF-36 at 3 and 6 months were lower in comparison to data available for Indian population (MCS: $51.68 \pm 5.5$, PCS: $47.87 \pm 8.17$ ). ${ }^{31}$ Poor SF- 36 at 3 and 6 months was found to be significantly associated with advancing age, poor baseline physical functional status, clinical frailty, and other ICU events like higher APACHE and SOFA scores, longer duration of mechanical ventilation, occurrence and duration of delirium, coma, pain, and usage of sedatives with or without analgesics.

Post-intensive care syndrome (PICS) is defined as new or worsening underlying impairment in physical, cognitive, or mental health status arising and persisting after hospitalization for critical illness. Understanding the epidemiology and risk factors is essential as the subsequent interventions to prevent PICS should be initiated at the earliest to prevent the cognitive impairment, improve mental, physical health and quality of life of ICU survivors, and reduce their long-term morbidity and mortality rates.

Our results highlight the importance of addressing cognitive impairment, mental health difficulties, and functional disabilities, which need to be monitored vigilantly. Since delirium is associated with long-term cognitive disability and functional limitations, interventions aimed at reducing delirium may help prevent brain injury associated with critical illness. Prevention of delirium with judicious use of sedative agents, following adequate pain management protocols, routine monitoring of delirium for all patients in ICU, and interventions like early mobilization and sleep protocols followed in ICU have been shown to mitigate the risk of delirium. However, it is unknown whether any preventive or treatment strategies can mitigate the risk of PICS, which needs further large-scale studies.

\section{Strengths and Limitations}

The strengths of our study include the availability of a follow-up cohort at 3 and 6 months. The attrition rate excluding the mortality was only $18.1 \%$, though mortality accounted for $28 \%$ of the missing cases at 6 months' follow-up. An important limitation of the current study was our inability to evaluate the patients' cognition before their current illness. We tried to address this limitation by excluding patients who were found to have severe dementia and preexisting cognitive dysfunction with a well-validated and objective Short IQCODE assessment tool. Another limitation of our study is that the questionnaires used were primarily validated for English-speaking population and hence application of such questionnaires and using their reference values for Indian population might not be a true representation of underlying cognitive dysfunction. The questionnaires were administered by intensivist with translation into Hindi in a subset of population, the validation of which is not available. We were not able to evaluate the risk of confounding by death or withdrawal during the study period. Finally, being an observational study, the risk of bias due to confounders not measured cannot be excluded.

\section{Conclusion}

In conclusion, cognitive impairment and decreased quality of life scores following critical illness is not uncommon and may persist. Baseline functional characteristics and various events occurring during intensive care admission may adversely affect the neurocognitive functions and quality of life of these post-ICU survivors. 


\section{References}

1. Needham DM, Davidson J, Cohen H, Hopkins RO, Weinert C, Wunsch H, et al. Improving long-term outcomes after discharge from intensive care unit: report from a stakeholders' conference. Crit Care Med 2012;40:502-509.

2. Ehlenbach WJ. The sobering reality of outcomes when older adults require prolonged mechanical ventilation. J Am Geriatr Soc 2014;62:183-185.

3. Hopkins RO, Weaver LK, Pope D, Orme JF, Bigler ED, Larson-LOHR V. Neuropsychological sequelae and impaired health status in survivors of severe acute respiratory distress syndrome. Am J Respir Crit Care Med 1999;160:50-56.

4. Jackson JC, Hart RP, Gordon SM, Shintani A, Truman B, May L,et al. Six-month neuropsychological outcome of medical intensive care unit patients. Crit Care Med 2003;31:1226-1234.

5. Ehlenbach WJ, Hough CL, Crane PK, Haneuse SJ, Carson SS, Curtis JR,et al. Association between acute care and critical illness hospitalization and cognitive function in older adults. JAMA 2010;303: 763-770.

6. Adhikari NK, Fowler RA, Bhagwanjee S, Rubenfeld GD. Critical care and the global burden of critical illness in adults. Lancet 2010;376: 1339-1346.

7. Chodosh J, Seeman TE, Keeler E, Sewall A, Hirsch SH, Guralnik JM,et al. Cognitive decline in high-functioning older persons is associated with an increased risk of hospitalization. J Am Geriatr Soc 2004; 52:1456-1462.

8. Rockwood K, Brown M, Merry H, Sketris I, Fisk J. Vascular Cognitive Impairment Investigators of the Canadian Study of Health and Aging. Societal costs of vascular cognitive impairment in older adults. Stroke 2002;33:1605-1609.

9. Jorm AF. The Informant Questionnaire on cognitive decline in the elderly (IQCODE): a review. Int Psychogeriatr 2004;16:275-293.

10. Katz S, Ford AB, Moskowitz RW, Jackson BA, Jaffe MW. Studies of illness in the aged. The index of Adl: a standardized measure of biological and psychosocial function. Jama 1963;185:914-919.

11. Pfeffer RI, Kurosaki TT, Harrah CH Jr, Chance JM, Filos S. Measurement of functional activities in older adults in the community. J Gerontol 1982;37:323-329.

12. Rockwood K, Song X, MacKnight C, Bergman H, Hogan DB, McDowell I, et al. A global clinical measure of fitness and frailty in elderly people. CMAJ 2005;173(5): 489-495.

13. Ferreira FL, Bota DP, Bross A, Melot C, Vincent JL. Serial evaluation of the SOFA score to predict outcome in critically ill patients. JAMA 2001;286:1754-1758.

14. Charlson ME, Pompei P, Ales KL, MacKenzie CR. A new method of classifying prognostic comorbidity in longitudinal studies: development and validation. J Chronic Dis 1987;40(5):373-383.

15. Sessler CN, Gosnell MS, Grap MJ, Brophy GM, O'Neal PV, Keane KA, et al. The Richmond Agitation-Sedation Scale: validity and reliability in adult intensive care unit patients. Am J Respir Crit Care Med 2002;166:1338-1344

16. Ely EW, Inouye SK, Bernard GR, Gordon S, Francis J, May L, et al. Delirium in mechanically ventilated patients: validity and reliability of the confusion assessment method for the intensive care unit (CAM-ICU). JAMA 2001;286:2703-2710.

17. Randolph C, Tierney MC, MohrE, ChaseTN. The Repeatable Battery for the Assessment of Neuropsychological Status (RBANS): preliminary clinical validity. J Clin Exp Neuropsychol 1998;20:310-319.

18. Ware JE. SF-36 Physical and Mental Health Summary Scales: a user's manual. Boston, MA: Health Assessment Lab, New England Medical Center, 1994.

19. Sharma A, Malhotra S, Grover S, Jindal SK. Incidence, prevalence, risk factor and outcome of delirium in intensive care unit: a study from India. Gen Hosp Psychiatry 2012;34:639-646.

20. Pandharipande PP, Girard TD, Jackson JC, Morandi A, Thompson JL, Pun BT, et al; BRAIN-ICU Study Investigators. Long-term cognitive impairment after critical illness. N Engl J Med 2013;369:1306-1316.

21. Jackson JC, Pandharipande PP, Girard TD, et al. Depression, posttraumatic stress disorder, and functional disability in survivors of critical illness in the BRAIN-ICU study: a longitudinal cohort study. Lancet Respir Med 2014;2(5):369-379. doi:10.1016/S22132600(14)70051-7

22. Sakusic A, O'Horo JC, Dziadzko M, Volha D, Ali R, Singh TD, et al. Potentially Modifiable Risk Factors for Long-Term Cognitive Impairment After Critical IIIness: A Systematic Review. Mayo Clin Proc 2018;93:68-82.

23. Hopkins RO, Suchyta MR, Snow GL, Jephson A, Weaver LK, Orme JF. Blood glucose dysregulation and cognitive outcome in ARDS survivors. Brain Inj 2010;24:1478-1484.

24. Van den Boogaard M, Schoonhoven L, Evers AW, van der Hoeven JG, van Achterberg T, Pickkers P. Delirium in critically ill patients: impact on long-term health-related quality of life and cognitive functioning. Crit Care Med 2012;40:112-118.

25. Jevtovic-Todorovic V, Absalom AR, Blomgren K, Brambrink A, Crosby G, Culley DJ, et al. Anaesthetic neurotoxicity and neuroplasticity: an expert group report and statement based on the BJA Salzburg Seminar. Br J Anaesth 2013;111:143-151.

26. Trzepacz PT. Is there a final common neural pathway in delirium? Focus on acetylcholine and dopamine. Semin Clin Neuropsychiatry 2000;5:132-148.

27. Dilger RN, Johnson RW. Aging, microglial cell priming, and the discordant central inflammatory response to signals from the peripheral immune system. J Leukoc Biol 2008;84:932-939.

28. Reidel B, Browne K, and Silbert B. Cerebral protection: inflammation, endothelial dysfunction, and post-operative cognitive dysfuncton. Curr Opin Anesthesiol 2014;27:89-97.

29. Hovens IB, Schoemaker RG, van der Zee EA, Heineman E, Izaks GJ, van Leeuwen BL. Thinking through postoperative cognitive dysfunction: How to bridge the gap between clinical and pre-clinical perspectives. Brain Behav Immun 2012;26:1169-1179.

30. Gunther ML, Morandi A, Krauskopf E, Pandharipande P, Giard TD, Jackson JC et al. The association between brain volumes, delirium duration, and cognitive outcomes in intensive care unit survivors: the VISIONS cohort magnetic resonance imaging study. Crit Care Med 2012;40:2022-2032.

31. Agarwal $R, D^{\prime} S$ Silva C. Assessment of quality of life in normal individuals using the SF-36 questionnaire. IJCCR 2017;3:43-47. 